\title{
Effects of the Oil-droplet Size and Entire Oil Fraction in Microcapsules on the Interior Oil Fraction
}

\author{
Kohshi KIKUCHI ${ }^{1}$, Shuichi YAMAMOTO ${ }^{2}$, Hirokazu SHIGA ${ }^{3}$, \\ Hidefumi YOSHII ${ }^{4}$, Shuji ADACHI ${ }^{5, \dagger}$ \\ ${ }^{1}$ Faculty of Liberal Studies, National Institute of Technology, Kumamoto College, \\ 2659-2 Suya, Koshi, Kumamoto 861-1102, Japan \\ ${ }^{2}$ Department of Applied Molecular Bioscience, Graduate School of Medicine, Yamaguchi University, \\ 2-16-1 Tokiwadai, Ube, Yamaguchi 755-8611, Japan \\ ${ }^{3}$ Department of Agriculture and Food Technology, Faculty of Bio-environmental Science, Kyoto Gakuen University, \\ 1-1 Nanjo-Otani, Sogabe, Kameoka, Kyoto 621-8555, Japan \\ ${ }^{4}$ Department of Applied Biological Science, Faculty of Agriculture, Kagawa University, \\ 2393 Ikenobe, Miki-cho, Kita-gun, Kagawa 761-0795, Japan \\ ${ }^{5}$ Division of Food Science and Biotechnology, Graduate School of Agriculture, Kyoto University, \\ Sakyo-ku, Kyoto 606-8502, Japan
}

\begin{abstract}
The interior oil fraction in a microcapsule, defined as the ratio of the volume of oil isolated from the microcapsule surface to the whole volume of the microcapsule, was statistically calculated based on a two-dimensional percolation model. As the entire oil fraction increased, the interior oil fraction first increased and then sharply declined after reaching a maximum value. It was demonstrated that reduction of the oil-droplet size was effective in the preparation of microcapsules with a high interior oil fraction.
\end{abstract}

Keywords: lipid microencapsulation, microcapsule, oil-droplet size ratio, percolation

\section{Introduction}

Much attention has been paid to the use of polyunsaturated lipids as functional food materials because of their physiological functionality [1]. However, the high susceptibility of lipids to oxidation due to their high level of unsaturation presents an obstruction to their use [2]. A potential method for suppressing or retarding lipid oxidation is microencapsulation into a dehydrated matrix [3-5]. Microencapsulation involves two steps: one is emulsification of lipid (or oil) with a dense aqueous solution of wall material, and the other is dehydration of the resultant oil-in-water $(\mathrm{O} / \mathrm{W})$ emulsion to prepare microcapsules. The entire oil content is designated as the volumetric fraction occupied with oil within the microcapsule, and the surface oil content of a microcapsule is defined as the fraction of oil exposed on the surface of the microcapsule to the entire oil content of the microcapsule. There is demand for encapsulating as much oil as possible within a microcapsule, in order to realize a higher entire oil fraction. However, the surface oil con-

(Received 3 Nov. 2015: accepted 21 Nov. 2015)

$\dagger$ Fax: +81-75-753-6285, E-mail: adachi@kais.kyoto-u.ac.jp tent is usually higher for microcapsules with higher entire oil fractions [6-8]. It has been demonstrated experimentally that the surface oil content was lower for an $\mathrm{O} / \mathrm{W}$ emulsion with smaller oil droplets $[9,10]$. We also investigated this fact using two- and three-dimensional models of percolation [11]. Because the oil exposed on the surface of the microcapsule is prone to oxidation, it would be desirable to prepare microcapsules that have a higher volumetric fraction of oil isolated from the surface. Such a fraction is designated here as an interior oil fraction.

In this study, the interior oil fraction was examined for microcapsules containing various entire oil fractions and oil-droplet sizes by simulation of a two-dimensional percolation model.

\section{Theoretical Considerations}

Although a microcapsule is a three-dimensional object and a three-dimensional percolation model is applicable, a two-dimensional percolation model was adopted in this study because of the limited memory of the personal computer used for simulation and the similarities between the two- and three-dimensional models of per- 
colation, which have been shown in our previous report [11]. A square was divided into $N \times N$ equal lattices, where $N$ was the number by which a side of the square was divided. The term $1 / N$ corresponded to the oil-droplet to microcapsule size ratio. The lattices occupied by oil were determined by the same statistical method as in our previous report [11]. Using the example shown in Fig. 1 where a side has been divided into 20 parts, the method is briefly explained. When a random number between 0 and 1 generated for a lattice was smaller than the entire oil fraction in a microcapsule, the lattice was regarded as being occupied by the oil and shaded in black or gray. The oil in the lattices shaded black was regarded as surface oil if the lattices were connected to one of the four sides of the square via the lattice side. On the other hand, the oil in the lattices shaded gray was isolated. The surface oil content was defined as the ratio of the number of the black-shaded lattices to the sum of the blackand gray-shaded lattices. In the case of Fig. 1, the content was calculated to be $38 / 133=0.286$. The entire oil fraction was calculated as the ratio of the number of the black- and gray-shaded lattices to all the lattices, that is, $(38+95) / 400=0.333$. The interior oil fraction was defined as the ratio of the number of the gray lattices to all the lattices, that is, $95 / 400=0.238$. The simulation was conducted at least 250 times with specified conditions of the entire oil fraction and $N$ value, and the average values

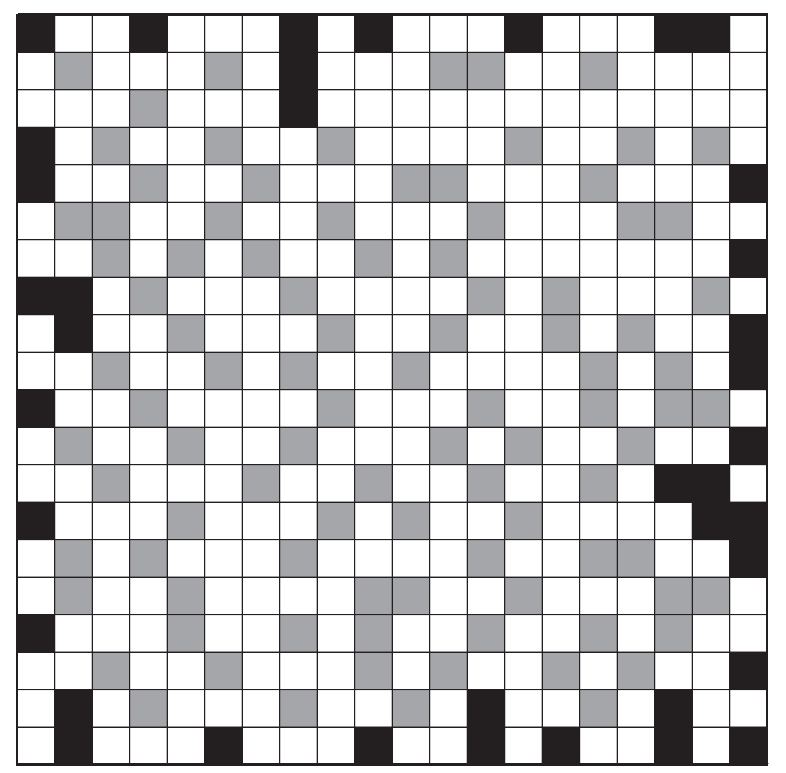

Fig. 1 Schematic of the two-dimensional model of percolation. One side of the square is divided into 20 parts. Lattices shaded in black and gray indicate the oil connected from the four sides of the square via the lattice side and the isolated oil, respectively. and standard deviations were calculated for the surface oil content and the interior oil fraction.

\section{Results and Discussion}

Figure 2 shows the surface oil contents and the interior oil fractions at various entire oil fractions for $N=50$ and 2500. The surface oil content was higher at a larger entire oil fraction, and it sharply increased at fractions larger than a certain value ( $c a .0 .4$ for $N=50,0.6$ for $N=$ 2500). As indicated in our previous study [11], the surface oil content became very low for a large $N$ value, that is, for small oil droplets. The interior oil fraction gradually increased, reached a maximum value, and then markedly declined to zero as the entire oil fraction became larger. The maximum value of the interior oil fraction was larger for $N=2500$ than $N=50$. The entire oil fraction at the maximum interior oil fraction was also larger for $N=2500$ than $N=50$. These facts indicated that the reduction of the oil-droplet size would enable us to prepare microcapsules in which a large amount of oil was isolated from the surface and would be protected from oxidation.

The effect of oil-droplet size, expressed by $1 / N$, on the maximum interior oil fraction was examined (Fig. 3). The surface oil content and the entire oil fraction at the maximum interior oil fraction are also shown in the figure. The maximum interior oil fraction became larger as $1 / N$ became smaller, that is, for smaller oil droplets in the microcapsule. The entire oil fraction and the surface oil

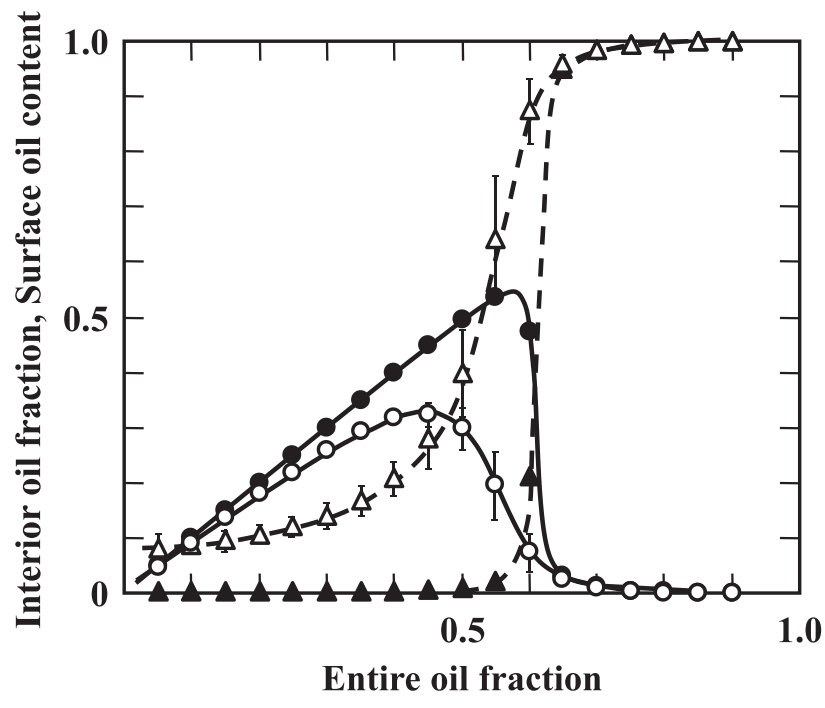

Fig. 2 Effects of the entire oil fraction on the surface oil content (triangles) and interior oil fraction (circles) for $N=50$ (open symbols) and $N=2500$ (closed ones). All the standard deviations are behind the symbols. 


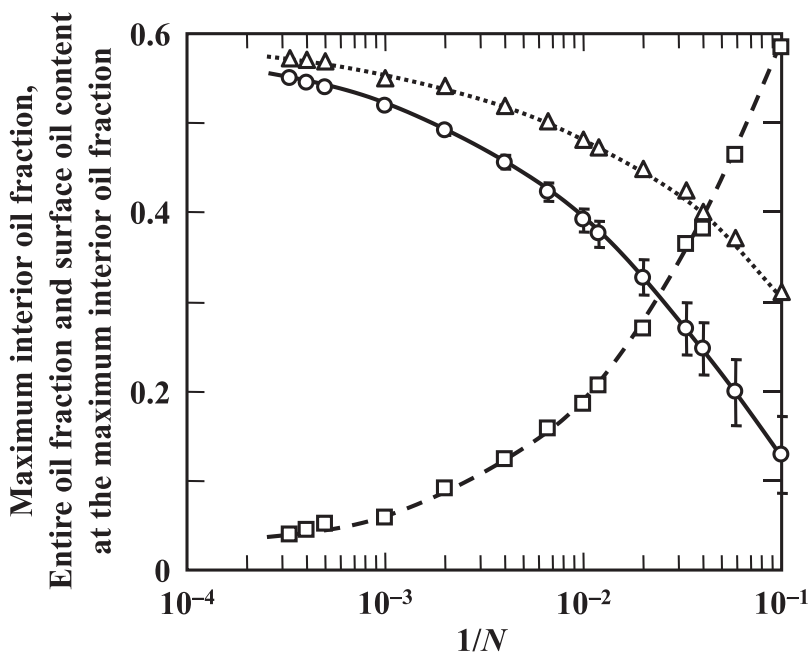

Fig. 3 The maximum interior oil fraction $(\bigcirc)$ for microcapsules with various oil-droplet sizes, expressed by the $1 / N$ values, and the entire oil fraction $(\triangle)$ and the surface oil content $(\square)$ at the maximum interior oil fraction. The vertical bar indicates the standard deviation. In many cases, these bars are behind the symbols.

content naturally showed similar and opposite dependencies, respectively, on the $1 / N$ value, as the maximum interior oil fraction. These results indicated that reduction of the oil-droplet size was advantageous for the preparation of a high entire oil content with low oxidation. However, excessive reduction in the oil-droplet size would not be so advantageous because the increase in the maximum interior oil fraction by reduction in $1 / N$ was not significant at smaller $1 / N$ values. The standard deviation in the maximum interior oil fraction was greater for larger $1 / N$ values, indicating that it would be difficult to prepare microcapsules with the same properties from large oil-droplets in a highly reproducible manner.

The above-mentioned simulations indicated that reduction of oil-droplet size was effective for the preparation of microcapsules with a high interior oil fraction. Excessive reduction of size was not necessary, and there was a critical entire oil content at which the interior oil fraction reached a maximum value, beyond which the interior oil fraction sharply decreased.

\section{Acknowledgement}

This study was partly supported by the Mishima Kaiun Memorial Foundation.

\section{References}

1) U. Gogus, C. Smith; n-3 Omega fatty acids: A review of current knowledge. Intl. J. Food Sci. Technol., 45, 417-436 (2010).

2) E. Choe, D. B. Min; Mechanisms and factors for edible oil oxidation. Compr. Rev. Food Sci. Food Safety, 5, 169-186 (2006).

3) N. Hardas, S. Danviriyakul, J. L. Foley, W. W. Nawar, P. Chinachoti; Accelerated stability studies of microencapsulated anhydrous milk fat. LWT-Food Sci. Technol., 33, 506513 (2000).

4) J. Velasco, M. C. Dobarganes, G. Márquez-Ruiz; Oxidation of free and encapsulated oil fractions in dried microencapsulated fish oils. Grasas Aceites, 51, 439-446 (2000).

5) K.-G. Wu, X.-H. Chai, Y. Chen; Microencapsulation of fish oil by simple coacervation of hydroxypropyl methylcellulose, Chinese J. Chem., 23, 1569-1572 (2005).

6) S. A. Hogan, B. F. McNamee, E. D. O’ Riordan, M. O’ Sullivan; Emulsification and microencapsulation properties of sodium caseinate/carbohydrate blends. Intl. Dairy J., 11, 137-144 (2001).

7) S. A. Hogan, B. F. McNamee, E. D. O’ Riordan, M. O’ Sullivan; Microencapsulating properties of sodium caseinate. J. Agric. Food Chem., 49, 1934-1938 (2001).

8) S. S. Umesha, B. Monahar, K. A. Naidu; Microencapsulation of $\alpha$-linolenic acid-rich garden cress seed oil: Physical characteristics and oxidative stability. Eur. J. Lipid Sci. Technol., 115, 1474-1482 (2013).

9) A. Soottitantawat, H. Yoshii, T. Furuta, M. Ohkawara, P. Linko; Microencapsulation by spray drying: Influence of emulsion size on the retention of volatile compounds. J. Food Sci., 68, 2256-2262 (2003).

10) S. M. Jafari, E. Assadpoor, B. Bhandari, Y. He; Nano-particle encapsulation of fish oil by spray drying. Food Res. Intl., 41, 172-183 (2008).

11) K. Kikuchi, S. Yamamoto, H. Shiga, H. Yoshii, S. Adachi; Surface oil content of microcapsules containing various oil fractions and oil-droplet sizes. Japan J. Food Eng., 14, 169173 (2013). 